\title{
A complex interplay between inflammation and immunity in liver cancer
}

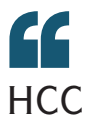

development

and tumour

burden were

markedly

reduced in IgA-

deficient mice

how that influences the establishment of liver cancer.

"Chronic inflammation is known to drive many cancers, especially liver cancer, and researchers have long thought that inflammation directly affects cancer cells, stimulating their division and protecting them from cell death," explains authors Michael Karin and Shabnam Shalapour. "We have now found that chronic liver inflammation also promotes cancer by suppressing immunosurveillance."

Previous work had shown that $\operatorname{IgA}^{+}$cells had immunosuppressive activity and interfered with the

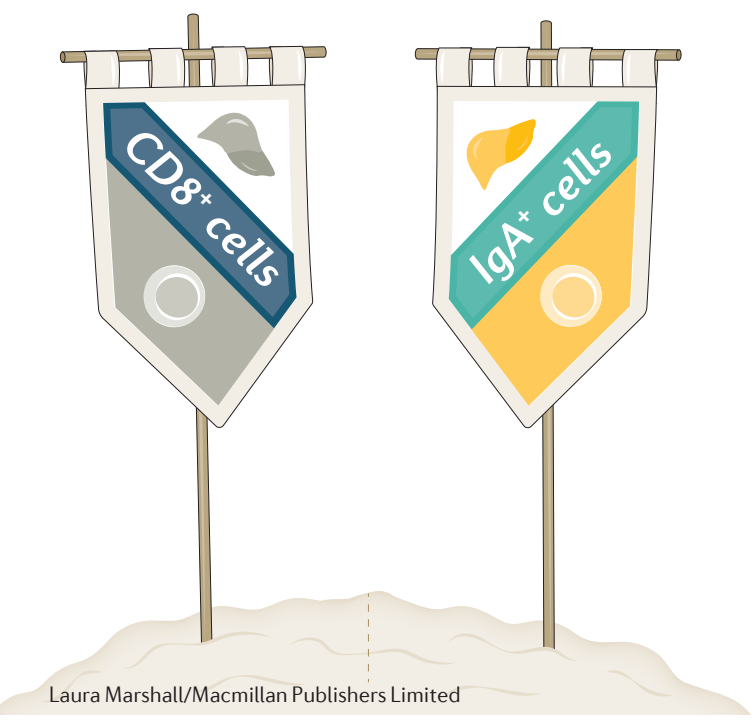

activation of CTLs. Moreover, patients with NASH and liver fibrosis had elevated circulating IgA levels compared with those without fibrosis. As such, the researchers sought to use a new mouse model of NASHinduced HCC (MUP-uPA mice fed a high-fat diet (HFD)) and liver samples from patients with NASH to study the role of $\operatorname{Ig} \mathrm{A}$ and $\operatorname{IgA}^{+}$cells in $\mathrm{NASH}$ and $\mathrm{NASH}$-induced HCC.

In two cohorts of patients with NASH $(n=598)$, serum IgA levels were elevated, which correlated with fibrosis score. The same trend was observed in mouse models of NASHdriven HCC that display fibrosis, including the HFD-fed MUP-uPA mice, but not in mouse models that were not fibrogenic. Both IgA $\mathrm{A}^{+}$cells and CTLs accumulated in mouse and human NASH-induced HCC samples. In mouse models, most $\operatorname{IgA}^{+}$cells expressed high levels of programmed cell death 1 ligand 1 (PDL1) and IL-10.

Examining the development of HCC in the MUP- $u P A$ mouse model, the investigators found that the mutational signatures in HCC in these mice were almost identical with those observed in human HCC. Importantly, $\operatorname{IgA}^{+}$cells and CTLs interplay in HCC development: HCC development and tumour burden were markedly reduced in IgAdeficient mice; by contrast, ablation or depletion of CTLs in IgA-deficient mice re-established HCC and absence of T and B cells accelerated HCC development to similar levels as Cd8a ablation.

Liver IgA $\mathrm{A}^{+}$cells were shown to directly suppress CTL activation in vitro and also inhibit CTL activation in vivo, inducing CTL exhaustion. Importantly, PDL1 blockade induced HCC regression in the MUP- $u P A$ model, reducing tumour load (with most large tumours having disappeared) and substantially decreasing liver IgA+IL- $10^{+}$cell abundance. Moreover, upon examination of the clonal expansion of liver CTLs from mice with HCC, PDL1 blockade was found to reactivate and expand antigen-specifc CTLs.

"In the battle between these two types of immune cells [IgA ${ }^{+}$cells and CTLs], immunosuppressive lymphocytes win - they use a molecule known as PDL1 to interfere with CTLs," says Karin and Shalapour. "With the brake on T cells, liver tumours formed and grew in the chronically inflamed mice."

"Our findings provide an explanation for the remarkable ability of so-called anti-PD1 drugs, which block the PDL1 receptor, to induce liver cancer regression," notes Karin and Shalapour, adding that the first member of this class of drugs, nivolumab, was approved by the FDA in 2017 for the treatment of advanced liver cancer.

The researchers are now exploring how immunosuppressive lymphocytes are recruited to the liver and are trying to determine the mechanism for their development. "This information may reveal a way to interfere with the recruitment or generation of these cells, which could provide new means for liver cancer prevention or early treatment," hopes Karin and Shalapour.

Katrina Ray

ORIGINAL ARTICLE Shalapour, S. et al. Inflammation-induced Ig $\mathrm{A}^{+}$cells dismantle anti-liver cancer immunity. Nature http:// dx.doi.org/10.1038/nature24302 (2017) 